Tropical Journal of Pharmaceutical Research January 2017; 16 (1): 219-224

ISSN: $1596-5996$ (print); 1596-9827 (electronic)

(c) Pharmacotherapy Group, Faculty of Pharmacy, University of Benin, Benin City, 300001 Nigeria.

All rights reserved.

Available online at http://www.tjpr.org

Original Research Article

http://dx.doi.org/10.4314/tjpr.v16i1.29

\title{
Clinical effect of Fuzheng quyu therapy in patients undergoing radiotherapy after cervical carcinoma surgery
}

\author{
Ming-Zhu Liu' ${ }^{\text {* }}$, Hao $\mathrm{Gu}^{2}$, Jin $\mathrm{Xu}^{3}$, Rui-Tai Fan ${ }^{2}$ and Xiao-Ming Deng ${ }^{1}$ \\ ${ }^{1}$ Department of Traditional Chinese Medicine, ${ }^{2}$ Department of Radiation Oncology, the First Affiliated Hospital of Zhengzhou \\ University, Zhengzhou, Henan, 450052, PR China, ${ }^{3}$ Encephalopathy, the First Affiliated Hospital of Henan University of \\ Traditional Chinese Medicine, Zhengzhou, Henan, 450000, PR China
}

*For correspondence: Email: Imzliumz@163.com

Received: 11 May 2016

Revised accepted: 7 December 2016

\begin{abstract}
Purpose: To examine the clinical effect of Fuzheng Quyu therapy in patients undergoing radiotherapy after cervical carcinoma surgery.

Methods: One hundred and twenty patients who underwent radiotherapy after cervical carcinoma surgery were selected randomly and divided evenly into control and study groups. Patients in the control group underwent radiotherapy only, whereas those in the study group received treatment designed to nourish healthy vital energy and eliminate blood stasis in addition to radiotherapy. Changes in the coagulation index of the two groups were recorded after treatment. The clinical effects and the incidence of adverse events were compared between the groups.

Results: The plasma prothrombin time and activated partial thromboplastin time improved after treatment in the study group to greater extents than in the control group. The levels of $D$-dimer, blood platelets and fibrinogen were lower after treatment in the study group than in the control group ( $p<$ 0.05). Improvement in Karnofsky Performance Score and quality of life score of the study group was superior to that of the control group. The proportion of patients who required drug intervention was much lower in the study group $(0.83 \%)$ than in the control group $(28.33 \%)(p<0.05)$. The incidence of adverse reactions was much lower in the study group (6.7\%) than in the control group (35.0\%, $p<$ 0.05).

Conclusion: Herbal therapy designed to nourish vital energy and eliminates blood stasis relieves highlevel blood coagulation, and also improves quality of life (QoL) in patients undergoing radiotherapy after cervical carcinoma surgery, thus suggesting its potential clinical applications.
\end{abstract}

Keywords: Fuzheng, Blood stasis, Cervical carcinoma, Radiotherapy, Quality-of-life

Tropical Journal of Pharmaceutical Research is indexed by Science Citation Index (SciSearch), Scopus, International Pharmaceutical Abstract, Chemical Abstracts, Embase, Index Copernicus, EBSCO, African Index Medicus, JournalSeek, Journal Citation Reports/Science Edition, Directory of Open Access Journals (DOAJ), African Journal Online, Bioline International, Open-J-Gate and Pharmacy Abstracts

\section{INTRODUCTION}

Cervical carcinoma is a frequent malignant disease in women that threatens both health and lives; its incidence is second only to that of breast cancer [1]. Currently, radiotherapy and surgical therapy are the standard treatments for patients with cervical carcinoma. However, some patients cannot tolerate radiotherapy, its duration is long, and it can cause various dangerous side effects and lower the quality of life (QoL) [2,3]. Studies [4,5] have indicated that radiation damages normal tissues while killing tumour cells, and it may induce short-term radiotherapy reactions and long-term complications. Moreover, some tumour cells are relatively insensitive to radiation, and the curative effect of radiotherapy may not be satisfactory, particularly in young patients with cervical carcinoma who require high postoperative QoL and hope to 
retain normal endocrine function and a sexual life, as radiotherapy can damage ovarian function. Hence, it is important to discuss alternative safe and effective therapies for cervical carcinoma.

Traditional Chinese Medicine (TCM) oncology has attracted increasing attention, and TCM plays an important role in the treatment of malignant tumours in females. Many clinical and experimental studies have demonstrated that TCM combined with radiotherapy or chemotherapy reduces toxicity and enhances efficacy $[6,7]$. Based on clinical practice and the characteristics of TCM oncology, this study developed a Chinese herbal compound to nourish vital energy and eliminate blood stasis following TCM dialectics and governmental ethical guidelines and evaluated its effect in patients undergoing radiotherapy, aiming to provide an empirical basis for its clinical application.

\section{METHODS}

This study was approved by the medical ethics committee of the First Affiliated Hospital of Zhengzhou University (approval no: LMZ20150615) and followed the Declaration of Helsinki [8]. Patients (120) who underwent radiotherapy after cervical carcinoma surgery at the First Affiliated Hospital, Zhengzhou University, Henan, China from May 2011 to May 2014 were randomly selected and divided into a study group and a control group ( $n$ $=60$ /group). Patients in the study group were aged $24-60$ years (mean, $30.20 \pm 1.20$ years); there were 54 cases of squamous carcinoma and 6 of adenocarcinoma. In the control group, patients were aged $25-62$ years (mean, $30.30 \pm$ 1.30 years); there were 56 cases of squamous carcinoma and four of adenocarcinoma. The general data showed no difference between the two groups $(p>0.05)$; thus, the results are comparable.

Patients included in the study had cervical carcinoma, underwent radiotherapy after cervical carcinoma surgery, had Karnofsky Performance Scores (KPSs) > 60, had at least 6 months' survival time, were aged 20 - 70 years, volunteered to participate in the study, and signed informed consent forms. Those who had severely impaired organ function or mental diseases, those who stopped taking the medicine or did not take the medicine for a sufficient time, and pregnant or nursing women were excluded.

Patients in the study group were treated with radiotherapy and Fuzheng Quyu therapy. The specific procedures were as follows. A linear accelerator was used for long-distance irradiation. The upper boundary was set at the $\mathrm{L}_{4-5}$ intervertebral disc, and the lower boundary at the inferior margin of obturator foramen; the space between the left and right pelvic bones was $1.5 \mathrm{~cm}$.

Irradiation was performed five times per week, at 2 Gy each time, for a total of $20-25$ treatments. Fuzheng Quyu preparation contained $30 \mathrm{~g}$ of Chinese yam, $25 \mathrm{~g}$ of Astragalus membranaceus, $25 \mathrm{~g}$ of Codonopsis pilosula, 20 $\mathrm{g}$ of Angelica sinensis, $20 \mathrm{~g}$ of Lycium barbarum, $15 \mathrm{~g}$ of Rehmannia glutinosa Libosch, $15 \mathrm{~g}$ of peony tree root bark, $15 \mathrm{~g}$ of rhizoma sparganii, one centipede, and one Buthus martensii Karsch. Different medicines were added according to clinical symptoms. White atractylodes, Poria cocos, and Schisandra chinensis were added for patients who showed a yellow facial complexion, abdominal distension, diarrhoea, and fatigue. Polygonatum odoratum and rhizoma anemarrhenae were added if patients had symptoms of dizziness, tinnitus, parched mouth and tongue, and insomnia. Colla corii asini and Caulis Spatholobi were added if patients were weak or fainted when they stood up. Those who felt cold in the limbs and had frequent micturition at night were given Curculigo orchioides and herba epimedii.

The medicine was decocted in water; $1000 \mathrm{~mL}$ of water was added during the first decoction, and patients took $100 \mathrm{~mL}$ of the resulting broth; 500 $\mathrm{mL}$ of water was added during the second decoction, and $100 \mathrm{~mL}$ of broth was taken. Ingredients for the broth were combined and mixed twice, and the broth was taken by patients twice daily (morning and evening) on the day of radiotherapy. The medication was stopped when radiotherapy was completed. Patients in the control group were treated with radiotherapy using the same treatment as those in the study group. Changes in routine blood indices were observed during treatment. Symptomatic treatment was administered for any abnormal condition.

\section{Study indices}

First was coagulation indicator. Fasting venous blood samples $(3 \mathrm{~mL})$ were collected in the morning, transferred to anticoagulant-containing tubes and centrifuged at 3,000 revolutions $/ \mathrm{min}$ for $10 \mathrm{~min}$. The yellow plasma (the upper layer) was collected and stored at $2-8{ }^{\circ} \mathrm{C}$. The activated partial thromboplastin time (APTT) was measured using the Compact BE fully automatic coagulometer. The D-dimer level was measured 
using a colloid gold immunofiltration assay. Platelet counts were determined using the Nanjing Pulang XFA9500 five-classification, fully automatic blood analyzer. Plasma prothrombin time (PT) was determined using the Beijing Precil C2000-1 semi-automatic coagulometer. Other data, including QOL scores and KPSs, were also recorded both before and after treatment. QOL was determined using a QOL scale, in which a higher score indicated a better QOL. KPS was determined using recognised scoring criteria, in which a higher score indicated a better physical condition. The symptomatic treatment outcomes were observed in the two groups. Adverse reactions after radiotherapy were recorded.

\section{Statistical analysis}

SPSS19.0 (SPSS, Inc., Chicago, IL, USA) was used to process the data. Data obtained before and after treatment were compared by paired ttest. Categorical data were compared by Chisquare tests. $P<0.05$ was considered statistically significant.

\section{RESULTS}

\section{Coagulation index}

After treatment, platelet and D-dimer levels were lower in the study than the control group (both $p$ values < 0.05). Also, both the APTT and PT of the study group were better than those of the control group (both $p$ values $<0.05$ ). No coagulation index improved significantly after treatment in the control group (all $p$ values > $0.05)$. All coagulation indices were markedly better in the study than in the control group (all $p$ values < 0.05) (Table 1).

\section{Comparison of clinical indices before and after treatment}

QoL score and KPS of the two groups did not differ before treatment; however, both scores improved significantly after treatment $(p<0.05)$. The improvement in the study group was significantly greater than that in the control group $(p<0.05$; Table 2).

\section{Symptomatic supportive treatment}

Significantly fewer patients underwent symptomatic supportive treatment in the study than in the control group $(p<0.01$, Table 3$)$, suggesting that the TCM therapy lowered the risk of radiotherapy-induced toxic side effects.

\section{Incidence of adverse events}

The incidence of adverse events in the study group $(6.70 \%)$ was much lower than that in the control group (35\%; $p<0.05$; Table 4).

The incidence of genitourinary tract and upper digestive tract adverse events in the study group was significantly lower than that in the control group $(p<0.05)$, but no difference in adverse events of the lower digestive tract or skin was observed between the groups, indicating that Fuzheng Quyu therapy effectively prevented radiation-induced adverse events in the genitourinary and upper digestive tracts, but was less effective for relieving radiation-induced adverse events in the lower digestive tract and skin.

Table 1: Coagulation index results for the two groups

\begin{tabular}{lccc}
\hline Group & & Study group & Control group \\
\hline \multirow{2}{*}{ APTT $(\mathrm{s})$} & Before treatment & $39.81 \pm 5.14$ & $38.78 \pm 5.11$ \\
& After treatment & $30.24 \pm 3.46^{* \#}$ & $35.79 \pm 4.99$ \\
PT $(\mathrm{s})$ & Before treatment & $17.43 \pm 3.16$ & $17.86 \pm 3.31$ \\
& After treatment & $12.8 \pm 2.53^{\text {\# }}$ & $16.42 \pm 2.74$ \\
D-dimer level $(\mathrm{mg} / \mathrm{L})$ & Before treatment & $0.69 \pm 0.47$ & $0.73 \pm 0.49$ \\
& After treatment & $0.52 \pm 0.48^{\text {\# }}$ & $0.67 \pm 0.48$ \\
Blood platelets $\left(\times 10^{9} / \mathrm{L}\right)$ & Before treatment & $295.72 \pm 100.24$ & $288.99 \pm 98.75$ \\
& After treatment & $268.69 \pm 96.87^{* \#}$ & $288.78 \pm 98.69$ \\
\hline
\end{tabular}

Note: ${ }^{\star} P<0.05$ compared with the level before treatment; ${ }^{\sharp} p<0.05$ compared with the control level

Table 2: Clinical indices before and after treatment (mean \pm standard deviation, $N=60$ )

\begin{tabular}{lcccc}
\hline \multirow{2}{*}{ Group } & \multicolumn{2}{c}{ QoL score } & \multicolumn{2}{c}{ KPS } \\
\cline { 2 - 5 } & Before & After & Before & After \\
\hline Study & $39.28 \pm 3.68$ & $41.48 \pm 3.49$ & $60.12 \pm 2.61$ & $75.67 \pm 2.85$ \\
Control & $39.34 \pm 3.33$ & $36.57 \pm 4.04$ & $60.21 \pm 2.53$ & $56.95 \pm 3.01$ \\
T & 0.067 & 4.994 & 0.090 & 14.131 \\
$P$-value & $>0.05$ & $<0.05$ & $>0.05$ & $<0.05$ \\
\hline
\end{tabular}


Table 3: Drug intervention rates $(N=60)$

\begin{tabular}{cccc}
\hline Group & $\begin{array}{c}\text { Undergoing } \\
\text { intervention }\end{array}$ & $\begin{array}{c}\text { Not undergoing } \\
\text { intervention }\end{array}$ & $\begin{array}{c}\text { Intervention rate } \\
(\%)\end{array}$ \\
\hline Study & 5 & 55 & 0.83 \\
Control & 17 & 43 & 28.33 \\
\hline
\end{tabular}

Table 4: Incidence of adverse events after radiotherapy

\begin{tabular}{lccccc}
\hline Group & $\begin{array}{c}\text { Genitourinary } \\
\text { tract }\end{array}$ & $\begin{array}{c}\text { LGI (including } \\
\text { pelvic cavity) }\end{array}$ & UGI & Skin & $\begin{array}{c}\text { Incidence of } \\
\text { untoward } \\
\text { reaction }\end{array}$ \\
\hline Study & 2 & 0 & 1 & 1 & $6.70 \%$ \\
Control & 10 & 1 & 8 & 2 & $35 \%$ \\
$X^{2}$ & 2.801 & 1.863 & 2.047 & 1.873 & 4.320 \\
$p$-value & $<0.05$ & $>0.05$ & $<0.05$ & $>0.05$ & $<0.05$ \\
\hline
\end{tabular}

LGl: lower gastrointestinal system; UGl: upper gastrointestinal system

\section{DISCUSSION}

Cervical cancer is commonly encountered in the clinic and is usually treated via surgery and radiotherapy. Many physicians, both in China and elsewhere, have evaluated the various treatments in detail. Roh et al [9] performed a prospective, randomized controlled study on 86 patients with cervical cancer who were randomly divided into a radical hysterectomy group (with pelvic nerve preservation) and a conventional radical hysterectomy group. Preservation of the cervical nerve improved the QoL. Zhou et al [10] randomly divided 67 patients with mid- and latestage cervical cancer into a group receiving three-dimensional conformal radiation therapy and chemotherapy and a group undergoing conventional radiotherapy and chemotherapy. The 3-year survival rates of the two groups were 87.10 and $61.10 \%$, respectively $(p=0.017)$.

Fabrini et al [11] found satisfactory outcomes for patients with stage lb cervival cancer who exhibited high-level risk factors after radiochemotherapy. Kim et al [12] reported that postoperative adjuvant radiochemotherapy was both more effective and more tolerable than was radiotherapy in patients with moderate risk factor levels after cervical cancer surgery. However, patients may experience different extents of impairment, including reduced immunity, aggravated blood loss, nausea and emesis, when treated with the therapies discussed above [13]. Hence, it is important to identify safer and more effective therapies.

Recently, Fuzheng Quyu (a TCM) has been shown to be of outstanding utility in patients undergoing chemotherapy after cervical cancer surgery. The principle of such therapy is that a disease should be treated by considering its origin. The treatment addresses both the symptoms and root causes of the disease, activating the blood, eliminating stasis, nourishing the spleen and tonifying the kidney [14]. With regard to the individual components of the prescription, Scutellaria baicalensis tonifies the spleen, increases vital energy, enhances immunity, activates lymphocyte phagocytosis and reduces the toxic side effects of radiotherapy.

Chinese yam imparts vital energy and renders the temperament more phlegmatic. A glycoprotein therein increases the rate of phagocytosis by white blood cells. Lycium barbarum $L$. nourishes the kidney and liver, removes heat, eliminates dryness, thoroughly scavenges free radicals, treats tissue impairment and improves immunity. Angelica sinensis relieves anemia by reducing the elimination of red blood cells. Rhizoma sparganii and Curcuma zedoary improve serum resistance to inflammation and infection. The combination of all of these herbs enhances patient recovery, improves immunity, regulates neural and endocrine functions, reduces adverse reactions and platelet aggregation, prevents fibrosis, kills tumor cells, increases blood flow and improves blood circulation.

Blood rheology indices of cancer patients differ significantly from those of normal control; the differences become more obvious as the disease progresses [15]. Cancer patients prefer chemotherapy to radiotherapy; however, chemotherapy cannot relieve hypercoagulation. TCMs that activate the blood and eliminate stasis can inhibit platelet aggregation, reduce blood viscosity, relieve hypercoagulation, correct blood 
stasis syndrome and improve QoL [16]. We found that after treatment, coagulation indices such as the D-dimer level and platelet count were much lower in the study group than in the control group. Moreover, both the APTT and PT also improved markedly in the study group, reflecting improvements in the hypercoagulation status and the extent of microcirculatory disturbance. Fuzheng Quyu given during postoperative chemotherapy also improved the Karnofsky and QoL scores and greatly reduced the incidence of toxic side reactions in the genitourinary and upper gastrointestinal tracts $(p$ $<0.05$ ). This may be because Fuzheng Quyu adjusts the internal environment, improves haematopoietic functions, enhances cellular immunity and relieves emesis and haemographic decline [17].

\section{Limitations of the study}

Although Fuzheng Quyu therapy improved the QOL in patients undergoing radiotherapy after cervical carcinoma surgery, relieved hypercoagulation and prevented and relieved toxic and side effects, the curative effects in only one disease case were evaluated as a result of time and other constraints. Thus, long-term observations and follow-up are lacking. These preliminary results suggest that Fuzheng Quyu therapy improves patient immunity, but the results need to be verified in further clinical investigations.

\section{CONCLUSION}

A herbal therapy designed to nourish vital energy and eliminate blood stasis relieves clinical symptoms, improves QoL and lowers the incidence of adverse events in patients undergoing radiotherapy after cervical cancer surgery. However, further clinical investigations before it can be recommended for actual clinical application.

\section{DECLARATIONS}

\section{Acknowledgement}

The authors thank all who supported this work.

\section{Conflict of Interest}

No conflict of interest associated with this work.

\section{Contribution of Authors}

The authors declare that this work was done by the authors named in this article and all liabilities pertaining to claims relating to the content of this article will be borne by them.

\section{Open Access}

This is an Open Access article that uses a funding model which does not charge readers or their institutions for access and distributed under the terms of the Creative Commons Attribution License (http://creativecommons.org/licenses/by 14.0) and the Budapest Open Access Initiative (http://www.budapestopenaccessinitiative.org/rea d), which permit unrestricted use, distribution, and reproduction in any medium, provided the original work is properly credited.

\section{REFERENCES}

1. Song $\mathrm{YH}$. Comparison of clinical and pathological characteristics of elderly and young and middle-aged patients with cervical carcinoma. Chin J Gerontol 2013; 33(22): 5600-5601.

2. Kowalik TF, DeGregori J,Leone $G$, Jakoi $L$ and Nevins JR. E2F1-specific induction of apoptosis and p53 accumulation, which is blocked by Mdm2. Cell Growth Differ 1998; (9): 113-118.

3. Plante M, Lau S, Brydon L, Swenerton K, LeBlanc R, Roy M. Neoadjuvant chemotherapy followed by vaginal radical trachelectomy in bulkystage IB1 cervical cancer: case report. Gynecol Oncol 2006; 101(2): 367-370

4. Liu Q, Liu ZS, Zhao RD, Zhao KK and Wang TJ. Curative effect of cisplatin sensibilization combined with radiotherapy and chemotherapy in treating middle and late cervical cancer. Chin J Gerontol 2014; (6): 47124714.

5. Liu YC, Liu YP. Pathogenesis and differential treatment of chemoradiotherapy induced injury. Acta Chin Med Pharmacol 2002; 30(4): 24.

6. Mell LK, Kochanski JD, Roeske JC, Haslam JJ, Mehta N, Yamada SD, Hurteau JA, Collins YC, Lengyel $E$ and Mundt AJ. Dosimetric predictors of acute hematologic toxicity in cervical cancer patients treated with concurrent cisplatin and intensity-modulated pelvic radiotherapy. Int J Radiat Oncol Biol Phys 2006; 66(5): 1356-1365

7. Hu DZ. Clinical observation of synergy and detoxification of Fuzheng anti-carcinoma prescription for advanced non-small cell lung cancer patients undergoing radiotherapy and chemotherapy. Shaanxi J Trad Chin Med 2005; 26(3): 209-210.

8. Declaration of Helsinki. The 59th World Medical Conference, 2008.

9. Roh JW, Lee DO, Suh DH, Lim MC, Seo SS, Chung J, Lee S, Park SY. Efficacy and oncologic safety of nervesparing radical hysterectomy for cervical cancer: a randomized controlled trial. J Gynecol Oncol 2015; 26(2): 90-99.

Trop J Pharm Res, January 2017; 16(1): 223 
10. Zhou YQ, Ma DY, Li XF, Tan BX. Clinical observation of late course three dimensional conformal radiotherapy combined with chemotherapy for locally advanced cervical cancer. Chin J Clin Oncol 2009; 36(1): 18-21.

11. Fabrini MG, Gadducci A, Perrone F, Cosio S, Laliscia C, Pasqualetti F, Grespi S, Cionini L. Clinical outcome of tailored adjuvant postoperative chemoradiotherapy in IB FIGO stage cervical cancer. Anticancer Res 2009; 29(10): 4205-4210.

12. Kim K, Kang SB, Chung HH, Kim JW, Park NH, Song YS. Comparison of chemoradiation with radiation as postoperative adjuvant therapy in cervical cancer patients with intermediate-risk factors. Eur J Surg Oncol 2009; 35(2): 192-196.

13. Parkin DM, Bray F, Ferlay J, Piisani P. Estimating the world cancer burden: Globocan 2000. Int J Cancer 2001; 94: 153-156.
14. Zeng $X$, Han $Y X, W u L X$. Significance analysis of the detection of HPV in cervicitis, cervical precancerous lesions and cervical cancer. Chin J Lab Diagn 2014; 18(1): 127-128.

15. Zhu H, Zhou ZX, Xue QH, Zhang X, He JH, Wang $L$. Treatment modality selection and prognosis of early stage small cell lung cancer: retrospective analysis from a single cancer institute. Eur J Cancer Care (Engl) 2013; 7(6): 1022-1025.

16. He J, Li CY, Li HS, He ZY, Yu XL. Effects of drug for invigorating blood circulation and eliminating stasis on blood platelet and blood rheology indexes of patients with non-small cell lung cancer in middle and advanced stages. Chin J Microcircul 2013; 23(3): 9-10,12.

17. Jia XB. The progress of young patients with cervical carcinoma. J Pract Obstetr Gynecol 2013; 29(3): 181 183. 